\title{
The in vitro genetics of barley (Hordeum vulgare L.): detection and analysis of reciprocal differences for culture response to 2,4-dichlorophenoxyacetic acid
}

W. Powell and

P. D. S. Caligari*
Scottish Crop Research Institute, Pentlandfield, Roslin, Midlothian EH25 9RF, Scotland.

The procedures of biometrical genetics have been used to detect and analyse reciprocal differences for the response of immature barley embryos to 2,4-dichlorophenoxyacetic acid in culture. Two genotypes were examined (4082 and R23) which had exhibited large reciprocal differences in culture in previous experiments. The $F_{1}, F_{2}$ and backcross generations, including full reciprocal families, were produced from these, true-breeding genotypes and evaluated in culture. The experiments confirmed the presence of reciprocal differences. Such differences were subsequently shown to stem from cytoplasmic effects alongside effects traceable to the mother tissue. The implications of these findings for the manipulation of barley genotypes in vitro are discussed.

\section{INTRODUCTION}

Genotypic differences exist in the response of cereals to in vitro culture and it has become apparent that a thorough understanding of the genetical control of growth and differentiation in such systems is necessary for the efficient use of cell culture technologies in crop improvement. In vitro selection (Bright, 1985), exploitation of somaclonal variation (Larkin, 1985) and the possibility of interspecific gene introgression (Armstrong, Nakamura and Keller, 1983) rely on the accessibility of commercially desirable cultivars for culture establishment and plant regeneration. A preliminary evaluation of culture response (Powell and Dunwell, 1987) may allow the identification of responsive genotypes and the transfer of desirable genes controlling culture response may be achieved by conventional methods. Knowledge of the genetical systems underlying culture response will complement this approach and may even be used to predict the response to selection (Mather and Jinks, 1982) and the probability of obtaining desirable genotypes from a given cross (Jinks and Pooni, 1976; Caligari, Powell and Jinks, 1985; Powell et al., 1985).

* Present address: Department of Agricultural Botany, Plant Sciences Laboratories, University of Reading, Whiteknights, Reading RG6 2AS, U.K
A number of factors may complicate the analyses of genotypic differences and in this paper attention is focused on a relatively neglected area of tissue culture response i.e., the possibility of reciprocal differences. A diallel analysis of in vitro response in barley indicated the presence of large reciprocal differences when immature embryos were cultured on media containing 2 , or more, $\mathrm{mg} \mathrm{l}^{-1}$ of 2,4-dichlorophenoxyacetic acid (Caligari, Powell and Goodall, 1987). The presence of reciprocal differences for culture response has also been reported in wheat (Lazar, Collins and Vian, 1983) and maize (Nesticky et al., 1983; Beckert and Qing, 1984; Tabata and Motoyoshi, 1965). In a more recent study (Tomes and Smith, 1985) on the initiation of embryogenic callus from maize genotypes, the reciprocal effects observed were attributed to segregating nuclear factors and explant source rather than cytoplasmic genetic factors. A distinction between maternal and cytoplasmic factors is important since the participation of the cytoplasm would imply that the organellar, cytoplasmic DNA is involved in the control of culture response. The objective of the present study was to investigate the underlying control of differences in culture response to the synthetic auxin 2,4-dichlorophenoxyacetic acid (2,4-D). This was achieved using biometrical genetical techniques which allowed not only the nuclear genetic 
basis to be quantified and analysed but also the cytoplasmic component.

\section{MATERIALS AND METHODS}

\section{Experimental material}

The parental material used in the present study consisted of the genetically multiply-marked spring barley stocks R23 and 4082. R23 possesses a range of morphological markers distributed among the seven pairs of homologous chromosomes with the alleles being in the dominant condition. 4082 is recessive at the corresponding loci. The parental, $F_{1}, F_{2}$ and backcross generations including full reciprocal families were produced in a glasshouse with a minimum 16-hour photoperiod at a temperature of $20^{\circ}-30^{\circ} \mathrm{C}$. Florets were emasculated by clipping the lemma and the palea and the anthers were removed using fine forceps (Pope, 1944). The sixteen families examined in the study are given in table 2 . In order to avoid problems associated with the effects of clipping, all the families sampled were clipped at anthesis.

\section{Culture of immature zygotic embryos}

Caryopses containing embryos 1-2 $\mathrm{mm}$ in length were removed from spikes representing each family. In the case of the six-row parent (4082) and segregants from crosses with it, only primary florets were excised. Caryopses were sterilised for 15 minutes in 2 per cent sodium hypochlorite with Tween 20 as a wetting agent. They were then washed in sterile distilled water. Embryos were excised and placed with the scutellum uppermost on $20 \mathrm{ml}$ of solidified medium (agar 0.7 per cent) at a frequency of five per petri-dish $(90 \times 15 \mathrm{~mm})$. The medium employed was based on that of Murashige and Skoog (1962) with the major salts at half the recommended concentration, 3 per cent sucrose and $4 \mathrm{mg} \mathrm{l}^{-1}$ of 2,4-D. Dishes were sealed with parafilm and incubated at $20^{\circ} \mathrm{C} \pm 2{ }^{\circ} \mathrm{C}$ with a 16-hour photoperiod produced by white fluorescent tubes $\left(30 \mu \mathrm{E} \mathrm{m}^{-2} \mathrm{~s}^{-1}\right)$.

After 28 days in culture, each embryo and attached callus was harvested. After blotting dry with paper tissues the fresh weight (F.Wt.) was recorded in mg. Following drying overnight at $35^{\circ} \mathrm{C}$, the dry weight (D.Wt.) was recorded and subsequently the water content (Wat) calculated.

\section{Experimental design and data transformation}

The experimental design was randomised complete blocks with six replicates (petri-dishes). Each family was represented by 5 embryos per petri-dish therefore giving a maximum of 30 embryos per family. Examination of the raw data revealed large positive correlations between the estimates of the means and variances. It was therefore considered necessary to transform the data. In practice it was found that taking the fourth root $\left(x^{1 / 4}\right)$ removed this scalar effect and all subsequent analyses were performed on the data transformed in this way (see Caligari et al., 1987).

\section{PRELIMINARY ANALYSES}

Analyses of variance were performed on the transformed data to test for differences between the 16 generation means for the characters: fresh weight (F.Wt.), dry weight (D.Wt.) and water content (Wat), and these are presented in table 1. It should be noted, however, that the three characters recorded here are not completely independent of one another since D.Wt. and Wat make up F.Wt. However, bearing this is mind, the analyses indicated that there were highly significant differences between families for the three characters scored. Furthermore, the interactions between replicates and families were also highly significant $(P<$ $0 \cdot 001$ ). The means and standard deviations for the three characters are given in table 2 . These statistics may be used to compare the family means as well as to detect and test for the presence of reciprocal differences. Student's " $t$-test" was applied and the values for " $t$ " from a number of comparisons of interest are given in table 3 . There were significant differences between the parents for the characters F.Wt. and Wat. The comparison between families 3 and 4 was also highly significant for these two characters indicating that there were large reciprocal differences between the responses of the $F_{1}$ hybrids in culture depending on the direction in which the cross was made. This reciprocal difference is presented visually in fig. 1; where immature embryo derived callus from the parental genotypes (R23 and 4082) together with the reciprocal $F_{1}$ hybrids are shown. It can be seen that the expression of the $F_{1}$ derived embryos in culture is clearly dependent on which direction the cross is made. Thus when 4082 is used as the female parent the resulting $F_{1}$ hybrid embryos are much more responsive in culture than those derived from the reciprocal. Apart from the comparison of family 3 with family 1 in table 3 , the rest of the comparisons measure reciprocal effects in the $F_{2}$ 's and backcrosses, and most proved to be significant. The fact that not all of them are significant indi- 
Table 1 Analyses of variance for the 16 families raised from the inbred lines R23 and 4082

\begin{tabular}{lcccc}
\hline Item & df & $\begin{array}{l}\text { F.Wt. } \\
\text { MS }\left(10^{2}\right)\end{array}$ & $\begin{array}{l}\text { D.Wt. } \\
\text { MS }\left(10^{2}\right)\end{array}$ & $\begin{array}{l}\text { Wat } \\
\text { MS }\left(10^{2}\right)\end{array}$ \\
\hline Reps & 5 & $12 \cdot 08^{*}$ & $16 \cdot 91^{* * *}$ & $13 \cdot 47^{* * *}$ \\
Families & 15 & $50 \cdot 65^{* * *}$ & $30 \cdot 12^{* * *}$ & $33 \cdot 49^{* * *}$ \\
Reps $\times$ Families & $72(3) \dagger$ & $8 \cdot 01^{* * *}$ & $6 \cdot 07^{* * *}$ & $6 \cdot 36^{* * *}$ \\
Error & $350(34) \dagger$ & $3 \cdot 11$ & 2.49 & $2 \cdot 37$ \\
\hline
\end{tabular}

$\dagger$ The number of missing observations.

* $P=0.05-0.01 ;{ }^{* * *} P=<0.001$.

cates that the cause(s) of such effects may not be simple and warrant further examination.

The comparison between families 3 and 1 is, in fact, the comparison of the higher scoring $F_{1}$ with the better parent and thus tests for heterosis, although it should be noted that it is not formally independent of the comparisons between 1 and 2 as well as 3 and 4 . There are no significant levels of heterosis observed, although the comparison for Wat approaches significance. There is, therefore no evidence, in this study, for superiority of the $F$, hybrid over the better scoring parent, for the characters examined.

\section{GENETICAL ANALYSES}

The presence of reciprocal differences has been detected in a number of tissue culture studies and these differences may be due to cytoplasmic inheritance and/or maternal effects. Cytoplasmic inheritance would result in persistent reciprocal differences and is the result of unequal contribu- tions of cytoplasmic determinants from the male and female gamete to the zygote (Jinks, 1964). This type of inheritance is through the female parent which contributes by far the major part of the zygotic cytoplasm. Maternal or mother tissue effects are transient in nature in that they, by definition, change with the genotype of the mother. A distinction between these two types of inheritance is important since a cytoplasmic component would suggest that organellar DNA residing in the chloroplasts or mitochondria is influencing in vitro development. It is also important since the successful manipulation of the character would depend on understanding the underlying modes of transmission. In order to investigate the genetical reasons for the reciprocal differences, a more intensive investigation of the two inbred lines was necessary. By producing generations in addition to the $F_{1}$ 's, genetical models can be fitted which allow the cytoplasmic and maternal effects to be estimated and their adequacy in describing the observed differences to be tested.

Table 2 The means $(\bar{x})$ and standard deviations (S.D.) for each of the 16 families for three chharacters

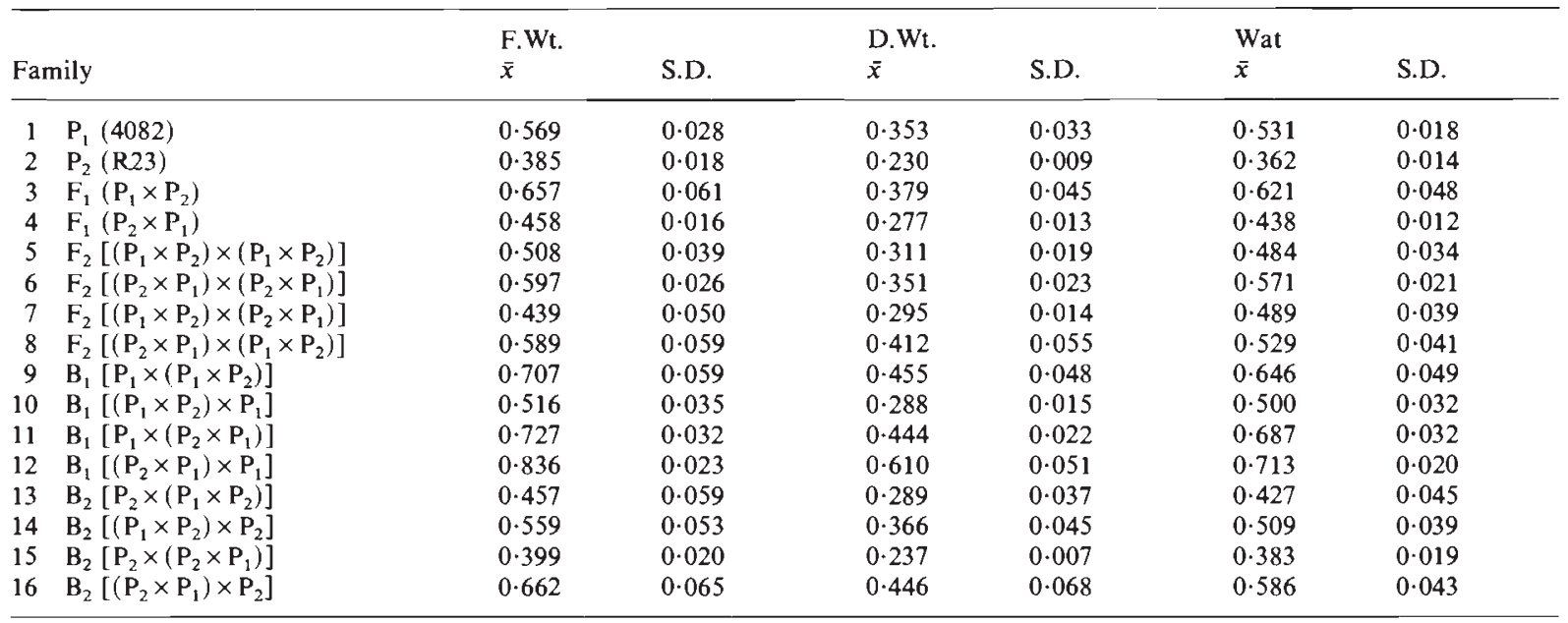




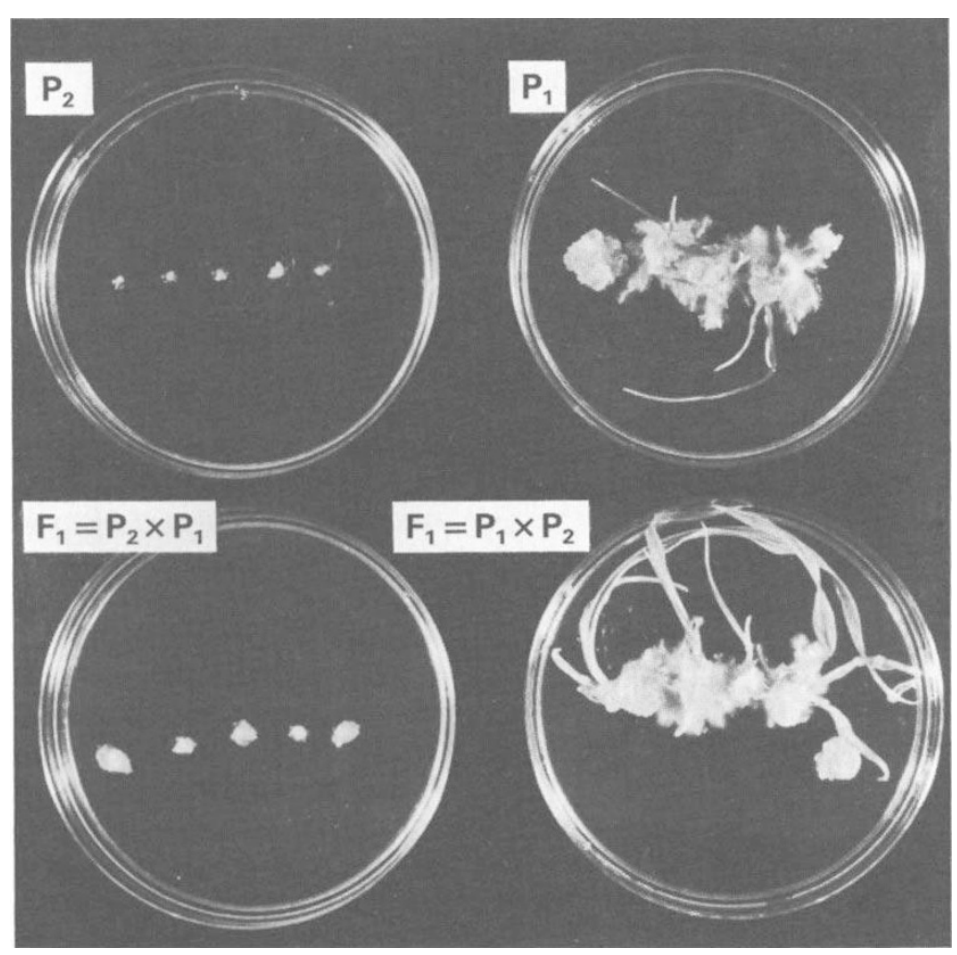

Figure 1 Response of immature barley embryos to medium containing $4 \mathrm{mg} \mathrm{I}{ }^{1}$ of $2,4-\mathrm{D}$. The two parents $\left(\mathrm{P}_{1}=4082\right.$ and $\left.\mathrm{P}_{2}=\mathrm{R} 23\right)$ are shown together with the reciprocal $F_{1}$ 's.

The expectations for the 16 families produced from the $F_{1}, F_{2}$ and backcrosses, including all possible reciprocals, are given in table 4 . The nuclear genetic contributions to the progeny means may be specified by the parameters: $m,[d],[h],[i],[j]$ and $[l]$, which provide estimates of the main effects and first-order interactions of these families (Mather and Jinks, 1982). The contribution of the cytoplasm is represented by [c] while the interactions between it and the additive, $[d]$, and dominance, $[h]$, nuclear determined effects are given as

Table 3 Student's " $t$-test" between the means of some of the 16 families

\begin{tabular}{|c|c|c|c|c|}
\hline \multicolumn{2}{|c|}{ Family comparison } & \multirow{2}{*}{$\begin{array}{l}\text { F.Wt. } \\
4 \cdot(6)^{* * *}\end{array}$} & \multirow{2}{*}{$\begin{array}{l}\text { D. Wt. } \\
3 \cdot 27^{* * *}\end{array}$} & \multirow{2}{*}{$\begin{array}{l}\text { Wat } \\
5 \cdot 02^{* * *}\end{array}$} \\
\hline 1 & vs. 2 & & & \\
\hline 3 & vs. 4 & $3.85^{* * *}$ & $2 \cdot 26^{*}$ & $4 \cdot 02^{* * * *}$ \\
\hline 3 & vs. 1 & $1 \cdot 59$ & 0.50 & 1.91 \\
\hline 5 & vs. 6 & $1 \cdot 77$ & $1 \cdot 00$ & $1 \cdot 88$ \\
\hline 7 & vs. 8 & $2 \cdot 40^{*}$ & $2 \cdot 36^{*}$ & $0 \cdot 75$ \\
\hline 9 & vs. 10 & $3 \cdot 27^{* *}$ & $3 \cdot 48^{* * *}$ & $2 \cdot 69 * *$ \\
\hline 11 & vs. 12 & $2 \cdot 52^{*}$ & $3 \cdot 37^{* * *}$ & 0.63 \\
\hline 13 & vs. 14 & $1 \cdot 41$ & $1 \cdot 27$ & $1 \cdot 32$ \\
\hline & vs. 16 & $4 \cdot 82^{* * *}$ & $4 \cdot 06^{* * *}$ & $4 \cdot 34^{* * *}$ \\
\hline
\end{tabular}

* $P=0.05-0 \cdot 01 ;$ ** $P=0.01-0 \cdot 001 ;{ }^{* * *} P=<0 \cdot 001$. $\left[c_{d}\right]$ and $\left[c_{h}\right]$ respectively. Parameters which represent the maternal components are also tabulated for two aspects: $\left[d_{m}\right]$, to represent the maternal effects traceable to the additive genetic component and $\left[h_{m}\right]$ that reflecting the dominance effects of the maternal genotype.

The model can be fitted to the observed data using weighted least squares with the inverse of the appropriate variance providing the weight for each family mean. The significance of each of the parameters estimated and the adequacy of the model can then be tested in the standard way (Mather and Jinks, 1982). The results of such model fitting are given in table 5 for the three characters scored. Models were considered to be satisfactory when the chi-square testing the goodness of fit was non-significant, and all the estimates of the parameters were themselves significantly different from zero. As can be seen from the table, the three characters give significant estimates for all the nuclear genetic parameters, apart from the additive genetic one. The lack of an additive genetic component, $[d]$, while the additive by additive, $[i]$, and the additive by dominance, $[j]$, interactions are significant, would indicate that there is dispersion of the alleles for increasing and 
Table 4 The expectations for the family means in terms of the genetic parameters for nuclear and cytoplasmic components as well as maternal effects

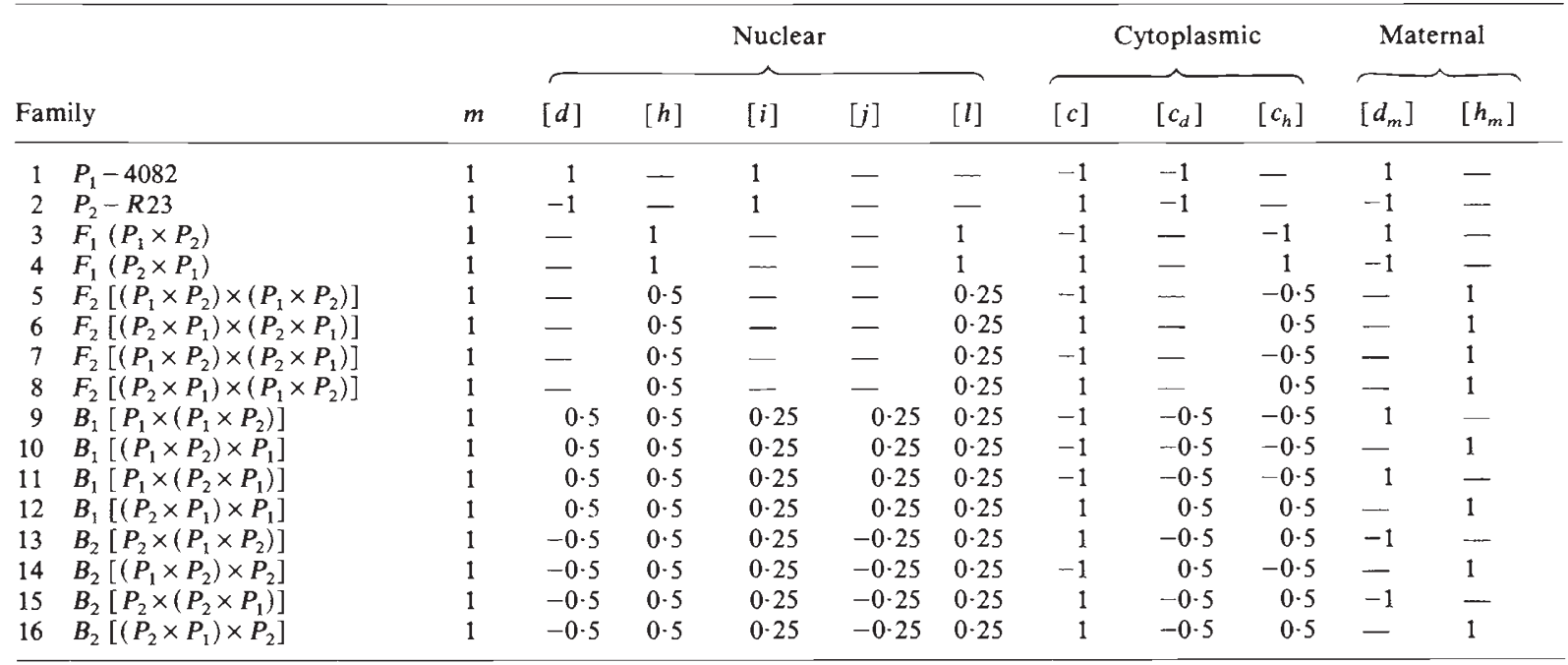

decreasing effect between the two parents (Jayasekara and Jinks, 1976).

The magnitudes and signs of $[h]$ and $[l]$ are not influenced by the way the alleles are distributed between the parents of the cross and hence these two components may be used to classify the predominant type of epistasis (non-allelic interaction), as described by Mather and Jinks (1982). In all three cases the $[h]$ increments of the majority of loci are positive while the $[l]$ increments for pairs of loci are negative. Epistasis is therefore mainly of the duplicate kind. This form of epistatis is generally associated with characters which have been exposed to directional selection whereas the dispersion of genes leading to a non-significant estimate of $[d]$ would suggest stabilizing selection. Although it is difficult to envisage a history of past selection directly affecting in vitro response, it is conceivable that the differences in these characters may be reflections of other morphological characters expressed in normal growth and development, and hence be subject to natural or artificial selection. In any case, the past history of selection and its effect could only be judged satisfactorily with a larger, and hence more representative, sample of genotypes. It should also be noted that the two genotypes used were chosen as representing extreme responses, yet they appear to show gene dispersion which at first sight appears paradoxical but, in fact, reflects the complex inheritance of these traits involving, as they do, reciprocal differences. Thus the parents have been chosen, as it turns out, more on the basis of their non-nuclear effects.

Table 5 Significant parameters in the model necessary to describe the differences between the families for the three characters

\begin{tabular}{|c|c|c|c|}
\hline & F.Wt. & D.Wt. & Wat \\
\hline$m$ & $0.236 \pm 0.095$ & $0.157 \pm 0.073$ & $0.307 \pm 0.085$ \\
\hline$[d]$ & - & - & 一 \\
\hline$[h]$ & $0.896 \pm 0.227$ & $0.564 \pm 0.176$ & $0.623 \pm 0.0204$ \\
\hline$[i]$ & $0.378 \pm 0.096$ & $0.282 \pm 0.083$ & $0.221 \pm 0.085$ \\
\hline$[j]$ & $0.192 \pm 0.060$ & $0.123 \pm 0.051$ & $0.162 \pm 0.054$ \\
\hline$[l]$ & $-0.572 \pm 0.141$ & $-0.386 \pm 0.115$ & $-0.400 \pm 0.128$ \\
\hline$[c]$ & $0.078 \pm 0.013$ & $0.059 \pm 0.011$ & $0.053 \pm 0.012$ \\
\hline$\left[c_{d}\right]$ & $0.135 \pm 0.029$ & $0 \cdot 146 \pm 0.030$ & $0.080 \pm 0.026$ \\
\hline$\left[c_{h}\right]$ & - & - & - \\
\hline$\left[d_{m}\right]$ & $0 \cdot 179 \pm 0.020$ & $0 \cdot 124 \pm 0 \cdot 016$ & $0.145 \pm 0.017$ \\
\hline$\left[h_{m}\right]$ & - & 一 & - \\
\hline$\chi_{[8]}^{2}$ & $6.8 \mathrm{n} . \mathrm{s}$ & $11 \cdot 0 \mathrm{n} . \mathrm{s}$ & $5 \cdot 0 \mathrm{n} . \mathrm{s}$ \\
\hline
\end{tabular}


There is evidence in table 5 for the presence of a cytoplasmic effect $[c]$ as well as its interaction, $\left[c_{d}\right]$, with the additive genetic component. There is also a significant maternal, $\left[d_{m}\right]$, component detected. It is interesting to note from the signs of the estimates of $[c]$ and $\left[d_{m}\right]$ and the signs attributed to them in the model (table 4 ) that they are, in fact, opposing one another in the two parents, with 4082 showing the increasing effect of the maternal component but a decreasing one for the cytoplasm while R23 shows, of course, the reverse. It would thus seem that as far as nuclear genes as well as reciprocal differences (of the two types) the parents used are not extremes, although 4082 is very responsive compared with other lines tested (Caligari et al., 1987).

\section{DISCUSSION}

The procedures of biometrical genetics have been used to elucidate the inheritance in response of immature barley embryos to in vitro culture. Such procedures showed that nuclear genes were involved and allowed the detection and separation of cytoplasmic and maternal effects. Indeed, they allowed all the components involved to be quantified within a single model, its adequacy to be tested statistically and the significance of the parameters to be assessed. Thus the methods outlined by Jinks, Perkins and Gregory (1972) for the analysis of reciprocal differences have allowed the response to be described although it is clearly complex in showing an array of underlying determinants.

Mathias, Fukui and Law (1986) using cytoplasmic substitution lines demonstrated the importance of cytoplasmic and nuclear-cytoplasmic interactions on tissue culture response in wheat. Calli were found to differ in their growth rates, their sensitivity to 2,4-D and their ability to organize shoot primordia. These authors do not, however, rule out the possibility that the cytoplasm may be influencing the original explant and not operating directly on culture response. In the present study on barley it is shown that both the cytoplasm and the maternal or mother tissue significantly effects culture response. The hexaploid nature of cultivated wheat has also allowed chromosome engineering techniques (Mathias and Fukui, 1986) to identify the role of specific chromosomes on tissue culture response in wheat. The substitution of the "Capelle" 4B chromosome resulted in an increase in cell proliferation and shoot regeneration.

Although the present study failed to detect significant positive heterosis it has previously been reported for callus growth in alfalfa (Keyes and Bingham, 1979) and in Nicotiana (Keyes et al., 1981). It has been recommended that hybrid vigour exhibited in cell and tissue culture may be of value in the identification of heterokaryons following somatic fusion. Smith, Rao and Combatti (1976) have demonstrated that partial selection for somatic fusion products of $N$. glauca and $N$. langsdorfii protoplasts may be based on the more rapid growth of hybrid colonies. However, the main emphasis must be on the need for a better understanding of the genetical basis for the expression of the character. It will then be possible to predict the level of heterosis likely to occur and to be clear about its underlying causation. This will then allow an assessment of its potential.

At present, advances in the application of the potential myriad of tissue culture techniques to commercially important crop species are hampered by the inability to hardle a sufficiently wide range of genetic material. A better understanding of the underlying genetical determination controlling in vitro response is therefore critical if the techniques are going to be developed as useful additions to those traditionally available for crop improvement. The multiply-marked stocks used in this study (R23 and 4082) differed in their in vitro response to 2,4-D. Both nuclear and cytoplasmic factors were clearly implicated in the control of culture response. It would therefore appear that the inheritance pattern, although complex, would still be capable of manipulation in conventional crossing programmes.

Acknowledgements We are grateful to V. Goodall for technical help and to Dr W. T. B. Thomas for the use of some computer programmes.

\section{REFERENCES}

ARMSTRONG, K. C., NAKAMURA, C. AND KELLER, W. A. 1983. Karyotype instability in tissue culture regenerants of Triticale (Triticosecale Wittmack) cv. "Welsh" from 6 months old callus culture. Z. Pflanzenzucht, 91, 233-245.

BFICKFRT, M. AND QING, C. M. 1984. Results of a diallel trial and a breeding experiment for in vitro aptitude in maize. Theor. Appl. Genet., 68, 247-251.

BRICilTT, S. W. I. 1985. Selection in vitro. In Bright, S. W. J. and Jones, M. J. K. (eds.) Cereal Tissue and Cell Culture, Martinus Nijhoff/Dr. W. Junk, pp. 232-260.

CALIGARI, P. D. S., POWELL, W. AND JINKS, J. L. 1985. The use of doubled haploids in barely breeding. 2. An assessment of univariate cross prediction methods. Heredity, 54, 353358.

CAligari, P. D. S., POWELL, W. ANI) GOODALL, V., 1987. The in vitro genetics of barley (Hordeum vulgare L.): Genetical analysis of immature embryo response to 2,4-dichlorophenoxyacetic acid. Heredity, 59, 285-292. 
JAYASEKARA, N. E. M. AND JINKS, J. L. 1976. Effects of gene dispersion on estimates of components of generation means and variances. Heredity, 36, 31-40.

JINKS, J. L. 1964. Extrachromosomal Inheritance, Prentice-Hall, London.

JINKS, J. L., PERKINS, J. M. AND GREGORY, S. R. 1972. The analysis and interpretation of differences between reciprocal crosses of Nicotiana rustica varieties. Heredity, $28,363-377$.

JINKS, J. L. AND POONI, H. S. 1976. Predicting the properties of recombinant inbred lines derived by single seed descent. Heredity, 36, 253-266.

KEYES, G. J. AND BINGHAM, E. T. 1979. Heterosis and ploidy effects on the growth of alfalfa callus. Crop Sci., 19,473-476.

KEYES, G. J., DEATON, W. R., COLLINS, G. B. AND LEGG, P. D. 1981. Hybrid vigour in callus tissue cultures and seedlings of Nicotiana tabacum L., J. Hered., 72, 172-174.

LARKIN, P. J. 1985 In vitro culture and cereal breeding. In Bright, S. W. J. and Jones, J. J. K. (eds) Cereal Tissue and Cell Culture, Martinus Nijhoff/Dr. W. Junk, pp. 274-296.

LAZAR, M. D., COLlinS G. B. AND VIAN, W. E. 1983. Genetic and environmental effects on the growth and differentiation of wheat somatic cell cultures. $J$. Hered., 74, 353-357.

MATHER, K. AND JINKS, J. L. 1982. Biometrical Genetics, 3rd edition. Chapman and Hall, London.

MATHIAS, R. J. AND FUKUI, K. 1986. The effect of specific chromosome and cytoplasm substitutions on the tissue culture response in wheat (Triticum aestivum) callus. Theor. Appl. Genet., 71, 797-800.
MATHIAS, R. J., FUKUI, K. AND LAW, C. N. 1986. Cytoplasmic effects on the tissue culture response of wheat (Triticum aestivum) callus. Theor. Appl. Genet., 72, 70-75.

MURASHIGE, T. AND SKOOG, F. 1962. A revised medium for rapid growth and bioassays with tobacco tissue cultures, Physiol. Plant., 15, 473-497.

NESTICKY, M., NOVAK, F. J., PIOVARCI, A. AND DOLEZELOVA, M. 1983. Genetic analysis of callus growth in maize (Zea mays L.) in vitro. $Z$. Pflantzenzucht., 91, 322-328.

POPE, M. N. 1944. Some notes on techniques on barley breeding. J. Hered., 35, 99-111.

POWELL, W., CALIGARI, P. D. S., MCNICOL, J. W. AND JINKS, J. L. 1985. The use of doubled haploids in barley. 3. An assessment of multivariate cross prediction methods. Heredity, 55, 249-254.

POWELL, W. AND DUNWELL, J. M. 1987. In vitro genetics of barley (Hordeum vulgare L.) 1. Response of immature embryos to 2,4-dichlorophenozyacetic acid. Heredity, (in press).

SMITH, H. H., RAO, K. N. AND COMBATTI, M. C. 1976. Interspecific hybridisation by protoplast fusion in Nicotiana: confirmation and extension. J. Hered., 67, 123-128.

TABATA, M. AND MOTOYOSHI, F. 1965. Hereditary control of callus formation in maize endosperm cultured in vitro. Japan J. Genet., 40, 343-355.

TOMES, D. T. AND SMITH, O. S. 1985. The effect of parental genotype on initiation of embryogenic callus from elite maize (Zea mays L.) germplasm. Theor. Appl. Genet., 70, 505-509. 